\title{
FIBROMA PRIMARIO DE TROMPA DE FALOPIO. REPORTE DE CASO
}

\author{
Elías Alexis Valladares Gutiérrez ${ }^{1, a}$, Christian Valeriano Alvarado ${ }^{1, b}$
}

\begin{abstract}
RESUMEN
Los fibromas de la trompa de Falopio son tumores benignos extremadamente poco frecuentes. La mayoría son asintomáticos y se encuentran incidentalmente. Frecuentemente son manejados con laparotomía. Se presenta un caso muy raro de fibroma extrauterino, dependiente del istmo de la trompa de Falopio izquierda en una mujer de 53 años; que tuvo los diagnósticos preoperatorios de probable fibroma de ovario y como segunda probabilidad mioma uterino parasitario y fue tratada por laparotomía con biopsia por congelación. La ecografía transvaginal mostró una masa compleja de bordes definidos a predomino sólido con componente quístico claramente separada del útero con flujo tumoral periférico e impedancia al flujo sanguíneo normal al estudio doppler; mientras que la tomografía espiral multicorte mostró una lesión expansiva sólida con componente quístico de 12,8 por $6,5 \mathrm{~cm}$ dependiente de anexo izquierdo. El nivel sérico del CA 125 y todas las otras pruebas de laboratorio estuvieron dentro de los límites normales. En el intraoperatorio y en la anatomía patológica no se encontró dependencia uterina alguna y la histología diagnosticó fibroma primario de trompa uterina.
\end{abstract}

Palabras clave: Fibroma; Trompa de Falopio(Fuente: DeCS BIREME).

\section{PRIMARY FALLOPIAN TUBE FIBROMA. CASE REPORT}

\begin{abstract}
Fibroids of the fallopian tube are benign tumors that are extremely rare. Most are asymptomatic and are found incidentally. They are often managed with laparotomy. We present a very rare case of an extrauterine fibroid, dependent on the isthmus of the left fallopian tube in a 53-year-old woman; who had the preoperative diagnoses of probable ovarian fibroma and as second probability uterine parasitic myoma and was treated by laparotomy with biopsy by freezing. The transvaginal ultrasound showed a complex mass of defined edges to predominantly solid with cystic component clearly separated from the uterus with peripheral tumor flow and impedance to normal blood flow to the Doppler study; while the multislice spiral tomography showed a solid expansive lesion with a cystic component of 12.8 by $6.5 \mathrm{~cm}$ depending on the left annex. The serum level of CA 125 and all other laboratory tests were within normal limits. In the intraoperative and in the pathological anatomy no uterine dependence was found and the histology diagnosed primary fibroma of the uterine tube.
\end{abstract}

Key words: Fibroma; Fallopian tube (Source: MeSH NLM).

\section{INTRODUCCIÓN}

Los fibromas son los tumores pélvicos benignos más comunes en mujeres en edad reproductiva ${ }^{1}$. Su prevalencia depende de la edad, y se pueden encontrar en casi el $80-90 \%$ de todas las mujeres a la edad de 50 años de edad ${ }^{2,3}$. Sin embargo, es muy probable que se subestime su verdadera incidencia, debido a no todos son sintomáticos. Muchos se detectan incidentalmente en los estudios de imágenes en mujeres asintomáticas, entre el 20 al $50 \%$ de las mujeres que los poseen son sintomáticas y es posible que deseen seguir un tratamiento ${ }^{4}$.
La bibliografía médica se refiere a ellos con diversos nombres: mioma, fibroma, fibromioma, leomioma, tumor fibroide, fibroleiomioma y leiomioma.

Los fibromas se originan en las células musculares lisas del útero (miometrio) o de las paredes vasculares que suministran al útero y su crecimiento depende de los estrógenos y la progesterona ${ }^{5,6}$.

El tumor primario de la trompa de Falopio es una entidad rara del tracto genital femenino ${ }^{7}$.

Instituto Nacional Materno Perinata

a Médico GínecoObstetra. Servicio de Emergencia, Departamento de Cuidados Críticos. Instituto Nacional Materno Perinatal. Magíster en Docencia e Investigación en Salud. Miembro titular de la Sociedad Peruana de Obstetricia y Ginecología. Especialista en Asesoría de Tesis. Lima-Perú. Código ORCID: 0000-0003-2789-4764

b Médico GínecoObstetra, subespecialista en Oncología Ginecológica, Servicio de Oncología Ginecológica. Instituto Nacional Materno Perinatal, Lima Perú.

Citar como: Valladares EA, Valeriano C. Fibroma primario de trompa de Falopio. Rev Peru Investig Matern Perinat 2019; 8(1):42-5.

DOI: https://doi.org/10.33421/inmp.2019142

Recibido: 07-03-19 Aprobado: 27-03-19 
Embriológicamente, el útero y la trompa se derivan en forma idéntica de los conductos de Müller, pero el fibroma es mucho más frecuentemente en el útero; aunque muy raramente, puede involucrar al ovario, ligamento ancho y las trompas de Falopio. Bajo la influencia de los esteroides sexuales como el estrógeno o progesterona, el miometrio uterino presenta cambios morfológicos y funcionales marcados durante el ciclo menstrual. La musculatura tubárica, por lo contrario, no muestra crecimiento significativo, incluso en el embarazo o la menstruación, hecho que podría explicar la diferencia en la incidencia entre los dos órganos.

El fibroma de la trompa de Falopio es una entidad ginecológica rara, la mayoría de casos son asintomáticos y excepcionalmente se hace el diagnóstico preoperatorio. La literatura señala que a menudo se tratan con laparotomía, y los estudios por imágenes preoperatorios los sospechan en pocos casos. El objetivo de este reporte es describir un caso de fibroma extrauterino primario de localización infrecuente.

\section{CASO CLÍNICO}

Mujer de 53 años de edad, grávida 3, para 3, acudió por dolor abdominal bajo y pélvico, intermitente de cuatro meses de evolución, de intensidad moderada y remisiones espontáneas. Ella había sido tratada con antibióticos por una probable enfermedad pélvica inflamatoria durante este período. Su historia no mostró antecedentes médicos importantes, no consumía fármacos de rutina y no tenía cirugías previas. Todos sus partos fueron normales sin intercurrencias en el período postparto. Al examen pélvico se detectó una tumoración en el anexo izquierdo con dolor leve a palpación y sin rebote. La ecografía transvaginal (DC8; Mindray Bio-Medical Electronics Co., Shenzhen, China) mostró en el anexo izquierdo una masa compleja de bordes definidos de 115,5 por $71,2 \mathrm{~mm}$, a predomino sólido con componente quístico de $37 \mathrm{x}$ $35,8 \mathrm{~mm}$, que podía distinguirse claramente separada del útero (Figura 1).

El estudio doppler mostró presencia de flujo tumoral periférico y el análisis de la forma de onda Doppler demostró impedancia al flujo sanguíneo normal (índice de pulsatilidad, IP =0,95; índice de resistencia, $\mathrm{IR}=$ 0,60 ) (Figura 2).

La tomografía espiral multicorte mostró una lesión expansiva sólida con componente quístico de 12,8 por $6,5 \mathrm{~cm}$ dependiente de anexo izquierdo. El nivel sérico del CA 125 fué $12,7 \mathrm{U} / \mathrm{mL}$. Todas las otras pruebas de laboratorio estuvieron dentro de los límites normales.

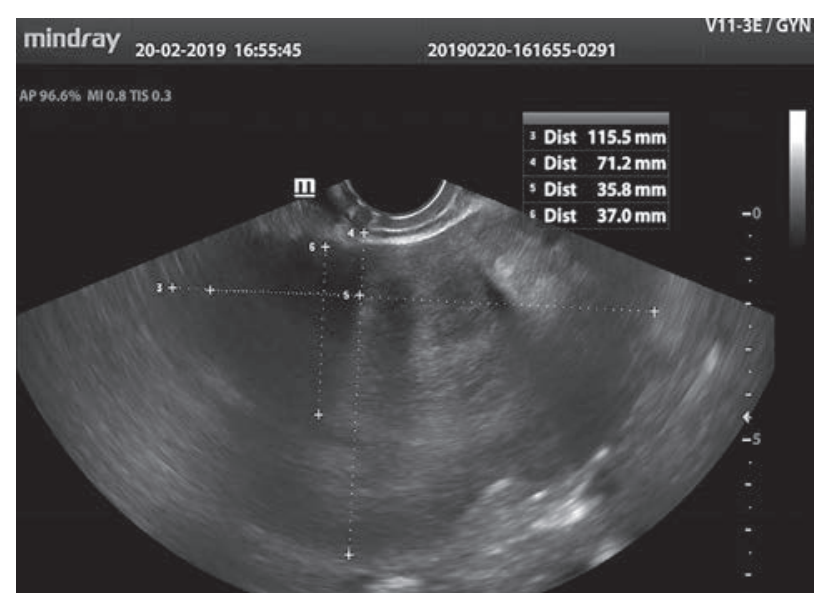

Figura 1. Masa compleja a predominio sólido de 115,5 por $71,2 \mathrm{~mm}$ localizada en el anexo izquierdo e independiente del útero.

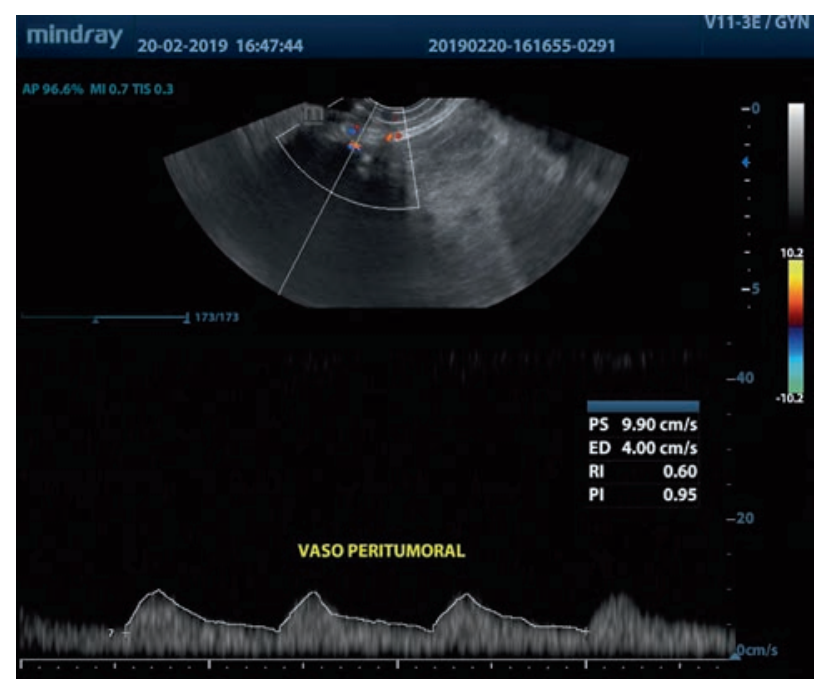

Figura 2. Análisis de la onda de flujo doppler que demuestra impedancia normal al flujo sanguíneo.

El diagnóstico preoperatorio incluyó probable fibroma de ovario o menos probable mioma uterino parasitario. Se planeó la resección quirúrgica del tumor más biopsia por congelación.

En la exploración quirúrgica, en el anexo izquierdo se encontró una tumoración ovoide sólida, con componente quístico, de superficie lisa, de 11 por 9 por 6 centímetros, pediculada, dependiente de la trompa de Falopio (Figura 3). Se encontró torsión del pedículo, el cual tenía siete vueltas completas. La biopsia por congelación concluyó negativo para neoplasia y la histopatología confirmó la presencia de un fibroma primario de trompa uterina. El postoperatorio cursó sin complicaciones y salió de alta al segundo día. Es de interés precisar que el dolor abdominal bajo y pélvico disminuyó dramáticamente después de la cirugía. 


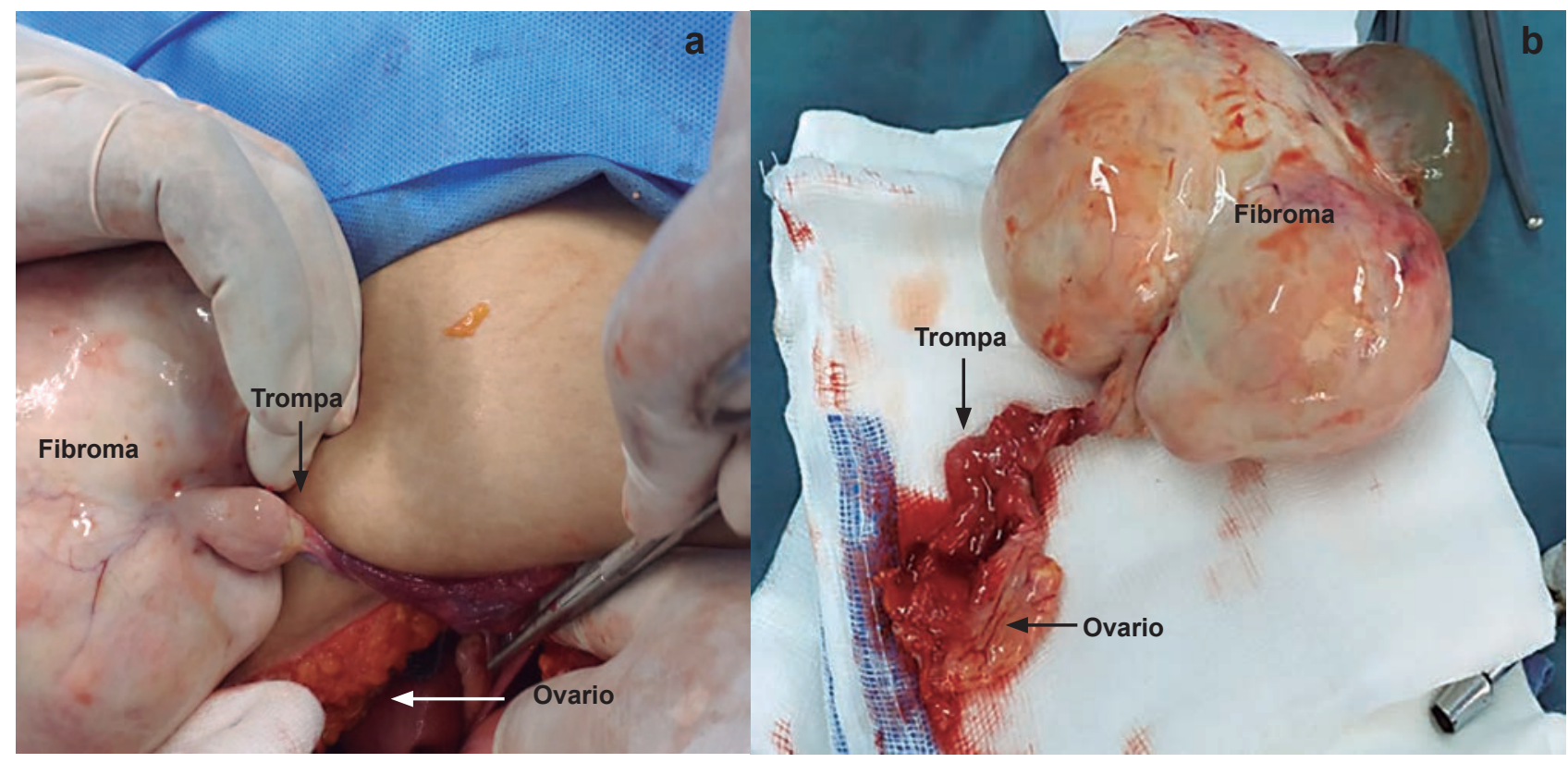

Figura 3. a)Trompa de Falopio izquierda conteniendo un fibroma de 11 por 9 por 6 centímetros en su porción ístmica. b) Hallazgos macroscópicos del tumor resecado, donde se evidencia el componente quístico y el pedículo torcido dependiente de la zona ístmica de la trompa de Falopio. Se aprecia la zona ampular y las fimbrias indemnes.

\section{DISCUSIÓN}

Existen escasos reportes de fibroma de la trompa de Falopio en la literatura y su incidencia se define como extremadamente rara $^{8-12}$. La mayoría de casos son asintomáticos y se encuentran incidentalmente al realizar una autopsia o una cirugía por otra indicación ${ }^{13}$. Cuando el fibroma progresa con crecimiento excesivo, torsión, cambios degenerativos o causa obstrucción, puede producir síntomas clínicos ${ }^{7}$; en el presente caso, el dolor intermitente que presentaba la paciente podría explicarse por el gran crecimiento del fibroma y por la torsión de siete vueltas completas que presentó su pedículo.

Los fibromas de la trompa se caracterizan por ser únicos y unilaterales ${ }^{7}$; lo que se confirma en este reporte. Adiferencia de lo señalado por Yang et al $^{9}$ de que los fibromas de la trompa de Falopio generalmente son nódulos pequeños; en este caso, el tumor fué considerablemente grande comparado con el tamaño de la trompa; similar al fibroma primario de trompa de 130 por 90 por $70 \mathrm{~mm}$ descrito por Ozkan et $a l^{14}$, Cisse et $a l^{10}$ y Takongmo et al ${ }^{15}$ también describieron haber encontrado fibromas de la trompa de Falopio "gigantes".

Se concuerda con lo reportado por la bibliografía que señala que la mayoría se localizan en el lado izquierdo ${ }^{7}$. La edad de la paciente en este reporte es mayor comparada con la edad media de 45,6 años descrita por Sikora-Szczęśniak et al ${ }^{16}$.

A pesar de excelentes imágenes de la pelvis con ultrasonido, tomografía computarizada o resonancia magnética que pueden ser útiles para diagnosticar esta condición, a veces existen dificultades para hacer un diagnóstico preciso ${ }^{17,18}$; y la laparoscopia puede ser usada para el diagnóstico definitivo y su manejo ${ }^{8}$. Sin embargo, a veces ni la laparoscopia ni el reconocimiento macroscópico pueden especificar la naturaleza del tumor, debiéndose hacer el diagnóstico final por histología.

Los fibromas de la trompa de Falopio se originan desde el miosalpinx o de las células de los vasos sanguíneos que irrigan la trompa. En la mayoría de casos se localizan en el istmo, raramente en la ámpula, pero también se ha encontrado como myoma nascens ${ }^{12}$. Al igual que los fibromas uterinos, los fibromas de la trompa de Falopio tienen localización submucosa, intramural y subserosa. Cuando son lo suficientemente grandes o se encuentran en statu nascendi tienden a torcerse o degenerar ${ }^{10,19,20}$.

En conclusión, Los fibromas de la trompa de Falopio son tumores benignos muy raros, siendo los fibromas sintomáticos como este que se presenta aún más raros, existiendo escasos reportes publicados en la literatura.

\section{Financiamiento: Autofinanciado}

Conflicto de interés: Los autores declaran no tener algún conflicto de intereses.

\section{REFERENCIAS BIBLIOGRÁFICAS}

1. De la Cruz MS, Buchanan EM. Uterine Fibroids: Diagnosis and Treatment. Am Fam Physician. 2017; 95(2):100-107.

2. Zimmermann A, Bernuit D, Gerlinger C, et al. Prevalence, symptoms and management of uterine fibroids: an international internet-based survey of 21,746 women. BMC Womens Health. 2012; 12(1):6. 
3. Grube M, Neis F, Brucker SY, Kommoss S, Andress J, Weiss $M$, et al. Uterine Fibroids - Current Trends and Strategies. Surg Technol Int. 2019; 18: 34.

4. Vilos GA, Allaire C, Laberge PY, Special contributors. The management of uterine leiomyomas. J Obstet Gynaecol Can. 2015;37(2): 157-181.

5. Bulun SE. Uterine fibroids. N Engl J Med. 2013; 369(14): 1344-1355.

6. Ishikawa $H$, Ishi $K$, Serna VA, et al. Progesterone is essential for maintenance and growth of uterine leiomyoma. Endocrinology. 2010;151(6): 2433-2442.

7. Chin-Chun Y,Kuo-Chang W, Pwu Ch, Peng-Hui W. Case Report. Primary Leiomyoma of the Fallopian Tube: Preoperative Ultrasound Findings. J Chin Med Assoc. 2007; 70(2):80-83

8. Wen KC, Yang CC, Wang PH. Primary fallopian leiomyoma manager by laparoskopy. J Minim Invasive Gynecol 2005; 12 : 193.

9. Yang $\mathrm{CC}$, Wen $\mathrm{KC}$, Chen $\mathrm{P}$, et al. Primary leiomyoma of the fallopian tube: preoperative ultrasound findings. J Chin Med Assoc. 2007; 70: 80-83.

10. Cisse M, Konate I, Dieng M, et al. Giant leiomyoma of fallopian tube: a rare aetiology of abdominal tumor. $J$ Gynecol Obstet Biol Reprod 2008; 37: 799-801.

11. Teymouri B, Sakhavar N, Mirteyomuri M. Leiomyoma of the fallopian tube: a case report and an unusual cause of abdominal pain. ZJRMS. 2008; 10: 159-162.

12. Sikora-Szczęśniak DL. Leiomyoma and leiomyoma cellulare of the fallopian tube: review of the literature and case reports. Menopause Rev. 2016; 15(3): 143-147.
13. Tomomaro Etoh1, Yoh Watanabe1, Izumi Imaoka2, Takamichi Murakami2 and Hiroshi Hoshiai1. Primary adenomyoma of the fallopian tube mimicking tubal malignant tumor. J Obstet Gynaecol Res. Vol. 38, No. 4: 721-723, April 2012

14. Ozkan Z, Gonen AN, Emir S, et al. Spontaneous ruprure of tubal leiomyoma causing haemoperitoneum. J Coll Physic Surg Pakistan 2014; 24 (Suppl 2): 91-92.

15. Takongmo S, Nkwabong E, Sando Z, et al. A case report of a giant fallopian tube leiomyoma mimicking a mesenteric tumor. Clin Mother Child Health (AJOL) 2010; 7: 1229-1231.

16. Sikora-Szczęśniak DL, Sikora W, Szczęśniak G. Leiomyoma cellulare in postoperative material: clinical cases. Med Stud 2013; 29: 144-151.

17. Jain RV, Case report: Fallopian tuve fibroid. MOJ Womens Health. 2017;5(1):189-190.

18. Ricci P, Troncoso JL, Contreras L, Contreras MS. Mioma primario de la trompa de Falopio: una localización muy infrecuente. Rev Chil Obstet Ginecol. 2013; 78 (4): 317- 319.

19. Fasih N, Prasad Shambhoue AK, Macdonald DB, Frasser-Hill MA, Papadatos D, Kielar AZ, et al. Leiomyomas beyond the uterus: unusual locations, rare manifestations. Radiographics. 2008; 28. 1931- 48.

20. Mohapatra P, Sahoo S, Behera RN. Myoma of the fallopian tube: a rare case. J Evolution Medic Dent Scienc. 2014; 3 : $14418-14420$

Correspondencia: Elías Alexis Valladares Gutiérrez Av. Las Gaviotas 420 - Chorrillos. Teléfono: 999962787. Correo electrónico: alexye02@hotmail.com 